\title{
The willow bud galler Euura mucronata Hartig (Hymenoptera: Tenthredinidae): one polyphage or many monophages?
}

\author{
T Nyman \\ Department of Biology, University of Joensuu, PO Box 111, FIN-80101 Joensuu, Finland
}

\begin{abstract}
The nematine sawfly Euura mucronata Hartig (Hymenoptera: Tenthredinidae) induces galls in the buds of over 30 willow species across the Holarctic region. This extensive host range is surprising, since the other Euura gallers are mostly monophagous; thus, the feeding habit of $E$. mucronata would represent a switch from monophagy to extreme polyphagy. Previous morphological studies have divided $E$. mucronata into separate species, but the feeding ranges of these species are unknown, and it is even doubtful whether multiple species really exist. To study whether or not $E$. mucronata consists of cryptic host-associated sibling species, an allozyme study was conducted using gallers collected from six willow species occurring in northern Fennos-
\end{abstract}

candia. Electrophoretic data from seven variable enzyme loci show that: (1) ' $E$. mucronata' probably comprises at least three species with restricted host ranges, but the species may not be completely reproductively isolated from each other; (2) the pattern of host use is not explained by the phylogeny of willows; (3) the pattern of host use is not concordant with the overall chemical similarity of the hosts; and (4) simple allopatric speciation does not appear to explain the host associations. Consequently, it is possible that reasons such as differences in host phenology, habitat, or morphology, are responsible for the limits in host use in the group.

Heredity (2002) 88, 288-295. DOI: 10.1038/sj/hdy/6800042

Keywords: allozymes; coevolution; Euura; host races; insect-plant interactions; speciation

\section{Introduction}

Phytophagous insects are commonly more or less restricted in their use of host plants; most species feed on one or a few host species, and the hosts are usually closely related (Jermy, 1984; Bernays and Graham, 1988). This restricted diet is usually attributed to constraints on the ability of the insects to adapt to the chemical defences of the potential host plants (Ehrlich and Raven, 1964; Futuyma and Keese, 1992). Restrictions in host use can also be caused by, for example, phenological differences between hosts (Smith, 1988; Butlin, 1990; Groman and Pellmyr, 2000; Filchak et al, 2000), differential predation or parasitism on hosts (Bernays and Graham, 1988; Crespi and Sandoval, 2000), or constraints on the information processing capacities of the insects (Levins and MacArthur, 1969; Janz and Nylin, 1997). The last reason could be important if some of the potential host plants can be confused with unsuitable hosts, which would lead to the evolution of an 'unnecessarily' narrow host range (Levins and MacArthur, 1969; Janz and Nylin, 1997).

The causes of host specificity are directly linked to the evolution of host shifts by monophagous insects. During a host shift the insect species must be at least oligophagous and, thus, it is likely that the direction of host shifts

Correspondence: T Nyman, Department of Biology, University of Joensuu, P.O. Box 111, FIN-80101 Joensuu, Finland.

E-mail:Tommi.Nyman@joensuu.fi

Received 8 May 2001; accepted 21 September 2001 is influenced by some of the aforementioned factors. For example, it has been suggested that a given insect species is more likely to colonize hosts that are chemically (Ehrlich and Raven, 1964; Becerra, 1997) or ecologically (Mardulyn et al, 1997) similar to the original host. On the other hand, specialist insects could speciate simply by tracking the speciation events in their hosts (Mitter and Brooks, 1983; Mitter et al, 1991). It has to be noted that if the new host is too similar to the original host, no speciation of the insect will result, but only an expansion of the insect's feeding range (Thompson, 1994; Groman and Pellmyr, 2000).

The interpretation of insect-plant relationships is complicated by the existence of cryptic host-associated sibling species or host races (Mayr, 1963; Diehl and Bush, 1984). The monophagous species occurring on different host plants may be morphologically almost indistinguishable, and only identifiable using molecular markers. Molecular methods have been used to identify sibling species or host races, for example, in beetles (Sturgeon and Mitton, 1986), flies (Condon and Steck, 1997; Berlocher, 1999), sawflies (Roininen et al, 1993; Herbst and Heitland, 1994), and moths (Emelianov et al, 1995; Groman and Pellmyr, 2000).

The purpose of the present study was to reveal whether the willow bud galler, Euura mucronata Hartig (Hymenoptera: Tenthredinidae) is one polyphagous species or a sibling species complex consisting of several monophagous species. E. mucronata belongs to the nematine sawfly gallers that induce galls on willows (Salix 
spp.). There are probably about 200 species of nematine gallers, and the species have been divided into three genera based on the type of gall that they induce: Phyllocolpa species induce leaf folds or rolls, Pontania species induce various leaf galls, and Euura species induce midrib, petiole, bud, and stem galls (Smith, 1970; Price and Roininen, 1993).

Most nematine gallers are very selective in their use of host species (Price and Roininen, 1993). An exception to this general pattern is the extremely polyphagous $E$. mucronata, which induces galls on over 30 willow species throughout the Holarctic region (Smith, 1970; Hartley, 1992; Price and Roininen, 1993; Kopelke, 1998, 1999; H Roininen and AG Zinovjev, unpublished data). This extensive range of hosts is even more surprising considering that the other Euura species are strictly monophagous (Smith, 1970; Kopelke, 1996). Thus, the feeding habit of E. mucronata would represent a switch from strict monophagy to polyphagy (Price and Roininen, 1993; Nyman et al, 2000).

It has long been suspected that E. mucronata is not a single species, but the recognition and description of separate species has proven to be difficult (see Zinovjev and Vikberg, 1998; Kopelke, 1998, 1999). The difficulties partly stem from the extreme size variation of E. mucronata, which is probably a result of different sized galls (Benson, 1958). Malaise (1920) described two bud-galling Euura species from Swedish Lapland: E. lanatae (on Salix lanata L.) and E. lappo (on S. lapponum L.). The females of these species can be separated based on the shape of the sawsheath and the coloration of the antennae (Malaise, 1920). Malaise also described a third variant or subspecies, E. lappo var. hastatae (on S. hastata L.), which is morphologically almost identical with E. lappo. Unfortunately, Malaise did not discuss the other willow species found in the same area, and later E. lappo and E. lappo var. hastatae were placed into synonymy with E. mucronata (Viitasaari and Vikberg, 1985). This was, however, apparently erroneous because E. lanatae and E. lappo should pupate and overwinter in the galls that they induce, whereas E. mucronata should overwinter on the ground (Zinovjev and Vikberg, 1998). Thus, over 80 years after Malaise's (1920) study, the status of the recognized species, and their host ranges, are unknown (Viitasaari and Vikberg, 1985; Zinovjev and Vikberg, 1998; Kopelke, 1998, 1999).

To study whether host-associated genetic differentiation can be found in E. mucronata, enzyme electrophoretic variation was analysed in bud gallers collected from six willow species occurring in northern Fennoscandia. The aim was to find allozyme markers for the identification of possible sibling species, and to study how the host associations fit the aforementioned hypotheses concerning the causes of host specificity and the evolution of host shifts in insect herbivores.

\section{Materials and methods}

\section{Sample collection and preservation}

Samples were collected in August 1998 from the vicinities of the Abisko research station $\left(68^{\circ} 21^{\prime} \mathrm{N}, 18^{\circ} 49^{\prime} \mathrm{E}\right)$ in Sweden and the Kilpisjärvi research station $\left(69^{\circ} 03^{\prime} \mathrm{N}\right.$, $20^{\circ} 48^{\prime} \mathrm{E}$ ) in Finland. The distance between the stations is about $120 \mathrm{~km}$, and both stations are located c. $250 \mathrm{~km}$ north of the Arctic Circle. The local habitats are very similar, treeless tundra highlands (fells) surrounded by low mountain birch (Betula pubescens Ehrh. ssp. czerepanovii (N. I. Orlova) Hämet-Ahti) forests with abundant willow undergrowth.

Current-year growth shoots having galled buds were collected from six willow species occurring in both areas: Salix lanata L., S. glauca L., S. lapponum L., S. phylicifolia L., S. myrsinifolia Salisb., and S. hastata L. Shoots were collected from over 30 individual clones per host species in each location. The leaves were removed, and the shoots were placed in plastic bags. After the larvae had emerged from the galls (c. 2-3 weeks), they were allowed to pupate in tissue paper placed in the bags. The bags were kept at $+1{ }^{\circ} \mathrm{C}$ until April 1999, after which they were taken to $+8^{\circ} \mathrm{C}$ for 2 days, and then to room temperature. The emerging adults were sexed and stored individually in Eppendorf tubes at $-80^{\circ} \mathrm{C}$. Five females and five males reared from each host species in both locations were inspected under a stereomicroscope to examine how their morphology corresponds to the types described by Malaise (1920).

\section{Electrophoresis}

In all, 435 females and 145 males were used for electrophoresis (Tables 1 and 2). Prior to electrophoresis, the samples were homogenized in $60 \mu$ l sample buffer (Ferguson, 1980). The tubes were centrifuged at 10000 rpm for 4 min, after which $45 \mu$ l of the supernatant was used in standard starch gel electrophoresis according to the protocol outlined in Vuorinen (1984). The horizontal $12 \%$ starch gels were cut into 2-mm thick slices and stained for specific enzymes. Buffer systems were as in Roininen et al (1993) and Nyman et al (1998).

Seven variable enzyme loci were scored: phosphoglucomutase (Pgm, EC 5.4.2.2.), malic enzyme ( $\mathrm{Me}$, EC 1.1.1.40), malate dehydrogenase (Mdh-2, EC 1.1.1.37), isocitrate dehydrogenase (Idh, EC 1.1.1.42), aspartate aminotransferase (Aat-2, EC 2.6.1.1), triose-phosphate isomerase (Tpi, EC 5.3.1.1), and glucose-6-phosphate isomerase (Gpi, EC 5.3.1.9). The following six loci were found to be monomorphic at the $99 \%$ level, and were thus excluded from the statistical analyses: phosphogluconate dehydrogenase (Pgdh, EC 1.1.1.44), glycerol-3-phosphate dehydrogenase (G3pdh, EC 1.1.1.8), lactate dehydrogenase ( $L d h, \mathrm{EC}$ 1.1.1.27), hexokinase (Hk, EC 2.7.1.1), fumarate hydratase (Fumh, EC 4.2.1.2), and malate dehydrogenase (Mdh-1, EC 1.1.1.37)

\section{Data analysis}

Haplodiploid sex determination in hymenopterans means that males are haploid and females diploid (Gauld and Bolton, 1988). Consequently, all statistical analyses were performed separately for males and females. Allele frequencies and sample sizes are presented in Tables 1 and 2. Deviations from Hardy-Weinberg equilibrium (in females) in individual loci were tested by the exact test of Guo and Thompson (1992) in the ARLEQUIN version 2.000 program (Schneider et al, 2000).

Population structure was first studied by clustering analyses using the TFPGA version 1.3 program (Miller, 1997). UPGMA clustering analyses were based on Nei's (1978) unbiased genetic distances and Reynolds et al's (1983) Coefficient of Coancestry distances. Cluster support was inferred by bootstrapping 1000 times over loci. 
Table 1 Allele frequencies in female Euura mucronata. Hosts are abbreviated by giving only the three first letters of the specific name (eg, S. lan = Salix lanata . Location abbreviations are: $\mathrm{K}=$ Kilpisjärvi, $\mathrm{A}=$ Abisko

Host (Location)

Locus Allele S. lan (K) S. lan (A) S. gla (K) S. gla (A) S. lap (K) S. lap (A) S. phy (K) S. phy (A) S. myr (K) S. myr (A) S. has (K) S. has (A)

\begin{tabular}{|c|c|c|c|c|c|c|c|c|c|c|c|c|c|}
\hline \multirow[t]{5}{*}{$M e$} & $\begin{array}{l}(n) \\
123\end{array}$ & $\begin{array}{c}38 \\
0.211\end{array}$ & $\begin{array}{c}33 \\
0.152\end{array}$ & $\begin{array}{c}38 \\
0.289\end{array}$ & $\begin{array}{c}40 \\
0.225\end{array}$ & $\begin{array}{c}39 \\
0.000\end{array}$ & $\begin{array}{c}37 \\
0.000\end{array}$ & $\begin{array}{c}36 \\
0.000\end{array}$ & $\begin{array}{c}\mathbf{2 8} \\
0.000\end{array}$ & $\begin{array}{c}37 \\
0.027\end{array}$ & $\begin{array}{c}36 \\
0.000\end{array}$ & $\begin{array}{c}37 \\
0.014\end{array}$ & $\begin{array}{c}34 \\
0.000\end{array}$ \\
\hline & 100 & 0.684 & 0.712 & 0.605 & 0.600 & 0.051 & 0.027 & 0.222 & 0.304 & 0.189 & 0.333 & 0.541 & 0.485 \\
\hline & 85 & 0.000 & 0.000 & 0.000 & 0.000 & 0.000 & 0.000 & 0.000 & 0.000 & 0.000 & 0.000 & 0.014 & 0.015 \\
\hline & 80 & 0.105 & 0.136 & 0.105 & 0.175 & 0.885 & 0.946 & 0.778 & 0.696 & 0.770 & 0.653 & 0.432 & 0.500 \\
\hline & 63 & 0.000 & 0.000 & 0.000 & 0.000 & 0.064 & 0.027 & 0.000 & 0.000 & 0.014 & 0.014 & 0.000 & 0.000 \\
\hline \multirow[t]{10}{*}{ Pgm } & $(n)$ & 38 & 34 & 38 & 40 & 40 & 37 & 36 & 28 & 37 & 36 & 37 & 34 \\
\hline & 132 & 0.303 & 0.544 & 0.382 & 0.463 & 0.000 & 0.000 & 0.000 & 0.000 & 0.000 & 0.000 & 0.027 & 0.015 \\
\hline & 121 & 0.000 & 0.000 & 0.000 & 0.000 & 0.000 & 0.000 & 0.000 & 0.000 & 0.014 & 0.042 & 0.000 & 0.000 \\
\hline & 119 & 0.000 & 0.000 & 0.013 & 0.000 & 0.913 & 0.878 & 0.000 & 0.000 & 0.000 & 0.000 & 0.000 & 0.000 \\
\hline & 100 & 0.474 & 0.456 & 0.526 & 0.525 & 0.000 & 0.027 & 0.944 & 0.982 & 0.919 & 0.944 & 0.946 & 0.985 \\
\hline & 90 & 0.053 & 0.000 & 0.079 & 0.000 & 0.075 & 0.068 & 0.000 & 0.000 & 0.014 & 0.000 & 0.000 & 0.000 \\
\hline & 75 & 0.000 & 0.000 & 0.000 & 0.000 & 0.000 & 0.000 & 0.028 & 0.018 & 0.000 & 0.000 & 0.014 & 0.000 \\
\hline & 72 & 0.026 & 0.000 & 0.000 & 0.000 & 0.013 & 0.027 & 0.028 & 0.000 & 0.054 & 0.014 & 0.014 & 0.000 \\
\hline & 57 & 0.145 & 0.000 & 0.000 & 0.000 & 0.000 & 0.000 & 0.000 & 0.000 & 0.000 & 0.000 & 0.000 & 0.000 \\
\hline & 65 & 0.000 & 0.000 & 0.000 & 0.013 & 0.000 & 0.000 & 0.000 & 0.000 & 0.000 & 0.000 & 0.000 & 0.000 \\
\hline \multirow[t]{5}{*}{$M d h-2$} & $(n)$ & 38 & 33 & 38 & 40 & 40 & 37 & 36 & 28 & 37 & 36 & 37 & 34 \\
\hline & 162 & 0.000 & 0.000 & 0.000 & 0.000 & 0.000 & 0.000 & 0.000 & 0.000 & 0.000 & 0.000 & 0.014 & 0.000 \\
\hline & 100 & 0.829 & 0.955 & 0.961 & 0.925 & 0.138 & 0.135 & 0.500 & 0.554 & 0.568 & 0.528 & 0.689 & 0.662 \\
\hline & 85 & 0.171 & 0.045 & 0.039 & 0.075 & 0.863 & 0.851 & 0.500 & 0.446 & 0.432 & 0.458 & 0.270 & 0.338 \\
\hline & 29 & 0.000 & 0.000 & 0.000 & 0.000 & 0.000 & 0.014 & 0.000 & 0.000 & 0.000 & 0.014 & 0.027 & 0.000 \\
\hline \multirow[t]{3}{*}{ Tpi } & $(n)$ & 38 & 34 & 38 & 40 & 40 & 37 & 36 & 28 & 37 & 36 & 37 & 34 \\
\hline & 100 & 1.000 & 1.000 & 1.000 & 1.000 & 1.000 & 1.000 & 0.986 & 0.946 & 1.000 & 0.958 & 1.000 & 1.000 \\
\hline & -76 & 0.000 & 0.000 & 0.000 & 0.000 & 0.000 & 0.000 & 0.014 & 0.054 & 0.000 & 0.042 & 0.000 & 0.000 \\
\hline \multirow[t]{6}{*}{$I d h$} & $(n)$ & 38 & 34 & 38 & 40 & 39 & 37 & 36 & 28 & 37 & 36 & 37 & 34 \\
\hline & 100 & 1.000 & 0.985 & 1.000 & 0.988 & 0.231 & 0.284 & 0.611 & 0.429 & 0.541 & 0.264 & 0.203 & 0.088 \\
\hline & 73 & 0.000 & 0.015 & 0.000 & 0.000 & 0.000 & 0.000 & 0.000 & 0.000 & 0.014 & 0.000 & 0.000 & 0.000 \\
\hline & 59 & 0.000 & 0.000 & 0.000 & 0.013 & 0.769 & 0.716 & 0.389 & 0.500 & 0.432 & 0.736 & 0.689 & 0.912 \\
\hline & 41 & 0.000 & 0.000 & 0.000 & 0.000 & 0.000 & 0.000 & 0.000 & 0.000 & 0.014 & 0.000 & 0.000 & 0.000 \\
\hline & 0 & 0.000 & 0.000 & 0.000 & 0.000 & 0.000 & 0.000 & 0.000 & 0.071 & 0.000 & 0.000 & 0.108 & 0.000 \\
\hline \multirow[t]{6}{*}{ Aat-2 } & $(n)$ & 36 & 33 & 37 & 40 & 32 & 34 & 33 & 25 & 33 & 32 & 32 & 30 \\
\hline & 130 & 0.000 & 0.000 & 0.000 & 0.000 & 0.000 & 0.015 & 0.000 & 0.000 & 0.000 & 0.000 & 0.000 & 0.000 \\
\hline & 115 & 0.000 & 0.000 & 0.000 & 0.000 & 0.016 & 0.000 & 0.015 & 0.000 & 0.000 & 0.000 & 0.000 & 0.000 \\
\hline & 100 & 1.000 & 1.000 & 0.973 & 1.000 & 0.953 & 0.897 & 0.606 & 0.780 & 0.561 & 0.750 & 0.859 & 0.883 \\
\hline & 76 & 0.000 & 0.000 & 0.000 & 0.000 & 0.016 & 0.000 & 0.000 & 0.000 & 0.000 & 0.000 & 0.000 & 0.000 \\
\hline & 60 & 0.000 & 0.000 & 0.027 & 0.000 & 0.016 & 0.088 & 0.379 & 0.220 & 0.439 & 0.250 & 0.141 & 0.117 \\
\hline \multirow[t]{4}{*}{$P g i$} & $(n)$ & 38 & 34 & 38 & 40 & 40 & 37 & 36 & 28 & 37 & 36 & 37 & 34 \\
\hline & 124 & 0.013 & 0.000 & 0.039 & 0.000 & 0.013 & 0.014 & 0.000 & 0.000 & 0.000 & 0.000 & 0.000 & 0.000 \\
\hline & 100 & 0.987 & 1.000 & 0.961 & 1.000 & 0.988 & 0.986 & 1.000 & 1.000 & 1.000 & 1.000 & 0.973 & 0.956 \\
\hline & 74 & 0.000 & 0.000 & 0.000 & 0.000 & 0.000 & 0.000 & 0.000 & 0.000 & 0.000 & 0.000 & 0.027 & 0.044 \\
\hline
\end{tabular}

Population structure in the subgroups formed by the clustering analyses was further studied by using ARLEQUIN version 2.000 (Schneider et al, 2000) to calculate Wright's (1978) hierarchical $F$ statistics according to the methods of Weir and Cockerham (1984); the $F$ statistics were used to infer the effect of host and location on the total genetic variation within the subgroups. The fixation indices were calculated without the individual level, and their statistical significance was inferred by a nonparametric permutation procedure with 10000 permutations (Excoffier et al, 1992).

\section{Results}

Statistically significant deviations from Hardy-Weinberg equilibria were found only at the $\mathrm{Me}$ locus in the S. lanata (Kilpisjärvi) sample $(P=0.017)$ and at the $M d h-2$ locus in the $S$. glauca (Kilpisjärvi) sample $(P=0.039)$; in both cases there was a deficiency of heterozygotes. However, neither of these deviations is statistically significant if the significance levels are adjusted using a sequential Bonferroni correction for multiple tests (Rice, 1989).

The UPGMA clustering analyses based on Nei's (1978) unbiased genetic distances indicate the presence of three well-defined and strongly supported groups both in males and females (Figure 1): one group is formed by the S. phylicifolia + myrsinifolia + hastata samples (Cluster 1), one of S. lanata + glauca samples (Cluster 2), and one of the two $S$. lapponum samples (Cluster 3). Essentially similar results were obtained using Reynolds et al's (1983) Coefficient of Coancestry distances, and the bootstrap values were approximately the same.

There is statistically significant population subdivision in females within Cluster $1\left(F_{\mathrm{ST}}=0.075, P<0.0001\right)$ (Table 3). Most of the differentiation results from differences between hosts $\left(F_{\mathrm{HT}}=0.057, P=0.025\right)$, but there is 
Table 2 Allele frequencies in male Euura mucronata. Abbreviations are as in Table 1

Host (Location)

Locus Allele S. lan (K) S. lan (A) S. gla (K) S. gla (A) S. lap (K) S. lap (A) S. phy (K) S. phy (A) S. myr (K) S. myr (A) S. has (K) S. has (A)

\begin{tabular}{|c|c|c|c|c|c|c|c|c|c|c|c|c|c|}
\hline \multirow[t]{6}{*}{$M e$} & $(n)$ & 22 & 21 & 12 & 10 & 10 & 13 & 9 & 12 & 8 & 9 & 8 & 10 \\
\hline & 123 & 0.273 & 0.048 & 0.333 & 0.200 & 0.000 & 0.000 & 0000 & 0.000 & 0.000 & 0.000 & 0.000 & 0.000 \\
\hline & 100 & 0.636 & 0.762 & 0.583 & 0.500 & 0.300 & 0.077 & 0.222 & 0.083 & 0.250 & 0.333 & 0.375 & 0.500 \\
\hline & 85 & 0.000 & 0.000 & 0.000 & 0.000 & 0.000 & 0.000 & 0.000 & 0.000 & 0.000 & 0.000 & 0.125 & 0.000 \\
\hline & 80 & 0.091 & 0.190 & 0.083 & 0.300 & 0.700 & 0.923 & 0.778 & 0.833 & 0.750 & 0.444 & 0.500 & 0.400 \\
\hline & 63 & 0.000 & 0.000 & 0.000 & 0.000 & 0.000 & 0.000 & 0.000 & 0.083 & 0.000 & 0.222 & 0.000 & 0.100 \\
\hline \multirow{8}{*}{ Pgm } & $(n)$ & 22 & 21 & 12 & 10 & 10 & 13 & 9 & 12 & 8 & 9 & 8 & 9 \\
\hline & 132 & 0.273 & 0.143 & 0.500 & 0.600 & 0.000 & 0.000 & 0.000 & 0.000 & 0.000 & 0.000 & 0.000 & 0.000 \\
\hline & 119 & 0.000 & 0.000 & 0.000 & 0.000 & 1.000 & 0.923 & 0.000 & 0.000 & 0.000 & 0.000 & 0.000 & 0.000 \\
\hline & 100 & 0.455 & 0.857 & 0.333 & 0.300 & 0.000 & 0.000 & 1.000 & 1.000 & 1.000 & 1.000 & 1.000 & 1.000 \\
\hline & 90 & 0.000 & 0.000 & 0.000 & 0.000 & 0.000 & 0.077 & 0.000 & 0.000 & 0.000 & 0.000 & 0.000 & 0.000 \\
\hline & 75 & 0.000 & 0.000 & 0.000 & 0.100 & 0.000 & 0.000 & 0.000 & 0.000 & 0.000 & 0.000 & 0.000 & 0.000 \\
\hline & 65 & 0.000 & 0.000 & 0.083 & 0.000 & 0.000 & 0.000 & 0.000 & 0.000 & 0.000 & 0.000 & 0.000 & 0.000 \\
\hline & 57 & 0.273 & 0.000 & 0.083 & 0.000 & 0.000 & 0.000 & 0.000 & 0.000 & 0.000 & 0.000 & 0.000 & 0.000 \\
\hline \multirow[t]{5}{*}{$M d h-2$} & $(n)$ & 21 & 21 & 12 & 10 & 10 & 13 & 9 & 12 & 8 & 9 & 8 & 11 \\
\hline & 162 & 0.000 & 0.000 & 0.000 & 0.000 & 0.000 & 0.000 & 0.000 & 0.000 & 0.000 & 0.000 & 0.125 & 0.000 \\
\hline & 146 & 0.000 & 0.000 & 0.000 & 0.000 & 0.000 & 0.077 & 0.000 & 0.000 & 0.000 & 0.000 & 0.000 & 0.000 \\
\hline & 100 & 0.905 & 0.905 & 0.917 & 0.900 & 0.000 & 0.154 & 0.444 & 0.667 & 0.375 & 0.556 & 0.750 & 0.727 \\
\hline & 85 & 0.095 & 0.095 & 0.083 & 0.100 & 1.000 & 0.769 & 0.556 & 0.333 & 0.625 & 0.444 & 0.125 & 0.273 \\
\hline \multirow[t]{3}{*}{$T p i$} & $(n)$ & 22 & 21 & 12 & 10 & 10 & 13 & 9 & 12 & 8 & 9 & 8 & 11 \\
\hline & 100 & 1.000 & 1.000 & 1.000 & 1.000 & 1.000 & 1.000 & 1.000 & 0.917 & 1.000 & 1.000 & 1.000 & 1.000 \\
\hline & -76 & 0.000 & 0.000 & 0.000 & 0.000 & 0.000 & 0.000 & 0.000 & 0.083 & 0.000 & 0.000 & 0.000 & 0.000 \\
\hline \multirow[t]{4}{*}{$I d h$} & $(n)$ & 22 & 21 & 12 & 10 & 10 & 13 & 9 & 12 & 8 & 9 & 8 & 10 \\
\hline & 100 & 1.000 & 1.000 & 1.000 & 1.000 & 0.300 & 0.000 & 0.333 & 0.583 & 0.375 & 0.222 & 0.500 & 0.000 \\
\hline & 59 & 0.000 & 0.000 & 0.000 & 0.000 & 0.700 & 1.000 & 0.667 & 0.417 & 0.500 & 0.778 & 0.500 & 1.000 \\
\hline & 0 & 0.000 & 0.000 & 0.000 & 0.000 & 0.000 & 0.000 & 0.000 & 0.000 & 0.125 & 0.000 & 0.000 & 0.000 \\
\hline \multirow[t]{4}{*}{ Aat-2 } & $(n)$ & 19 & 17 & 8 & 10 & 8 & 11 & 7 & 10 & 7 & 8 & 8 & 7 \\
\hline & 100 & 1.000 & 1.000 & 1.000 & 1.000 & 0.875 & 0.818 & 0.429 & 0.800 & 0.714 & 1.000 & 0.750 & 0.857 \\
\hline & 76 & 0.000 & 0.000 & 0.000 & 0.000 & 0.000 & 0.091 & 0.000 & 0.000 & 0.000 & 0.000 & 0.000 & 0.000 \\
\hline & 60 & 0.000 & 0.000 & 0.000 & 0.000 & 0.125 & 0.091 & 0.571 & 0.200 & 0.286 & 0.000 & 0.250 & 0.143 \\
\hline \multirow[t]{4}{*}{$P g i$} & $(n)$ & 22 & 21 & 12 & 10 & 10 & 13 & 9 & 12 & 8 & 9 & 8 & 11 \\
\hline & 124 & 0.000 & 0.048 & 0.000 & 0.000 & 0.000 & 0.000 & 0.000 & 0.000 & 0.000 & 0.000 & 0.000 & 0.000 \\
\hline & 100 & 1.000 & 0.952 & 1.000 & 1.000 & 0.900 & 1.000 & 1.000 & 1.000 & 1.000 & 1.000 & 1.000 & 1.000 \\
\hline & 89 & 0.000 & 0.000 & 0.000 & 0.000 & 0.100 & 0.000 & 0.000 & 0.000 & 0.000 & 0.000 & 0.000 & 0.000 \\
\hline
\end{tabular}

Table 3 Hierarchical $F$ statistics for each locus and averaged across all loci in female Euura mucronata in Cluster 1 (see Figure 1)

\begin{tabular}{|c|c|c|c|c|c|c|c|c|}
\hline & \multicolumn{8}{|c|}{ Locus } \\
\hline & $M e$ & $P g m$ & $M d h-2$ & Tpi & $I d h$ & Aat-2 & $P g i$ & Total \\
\hline$F_{\text {ST (OVERALL) }}$ & $0.074^{* * *}$ & 0.003 & 0.013 & $0.017^{*}$ & $0.156^{* * *}$ & $0.074^{* * *}$ & $0.016^{*}$ & $0.075^{* * *}$ \\
\hline $\begin{array}{l}F_{\mathrm{HT} \text { (HOSTS) }} \\
F_{\text {LH (LOCATIONS WITHIN HOSTS) }}\end{array}$ & $\begin{array}{l}0.083 \\
0.007\end{array}$ & $\begin{array}{r}0.005 \\
-0.001\end{array}$ & $\begin{array}{r}0.028 \\
-0.010\end{array}$ & $\begin{array}{r}-0.001 \\
0.019\end{array}$ & $\begin{array}{l}0.086 \\
0.092^{* * *}\end{array}$ & $\begin{array}{l}0.043 \\
0.040^{*}\end{array}$ & $\begin{array}{r}0.033 \\
-0.010\end{array}$ & $\begin{array}{l}0.057^{*} \\
0.030^{* * *}\end{array}$ \\
\hline $\begin{array}{l}F_{\text {LT (LOCATIONS) }} \\
F_{\text {HL (HOSTS WITHIN LOCATIONS) }}\end{array}$ & $\begin{array}{l}-0.029 \\
0.090^{* *}\end{array}$ & $\begin{array}{r}0.006 \\
-0.001\end{array}$ & $\begin{array}{r}-0.011 \\
0.020\end{array}$ & $\begin{array}{l}0.012 \\
0.010^{* *}\end{array}$ & $\begin{array}{l}0.040 \\
0.135^{* * *}\end{array}$ & $\begin{array}{l}0.015 \\
0.065^{* *}\end{array}$ & $\begin{array}{r}-0.011 \\
0.023\end{array}$ & $\begin{array}{l}0.004 \\
0.073^{* * *}\end{array}$ \\
\hline $\begin{array}{l}F_{\text {HK (HOSTS IN KILPISJÄRVI) }}{ }^{a} \\
F_{\text {HA (HOSTS IN ABISKO) }}{ }^{a}\end{array}$ & $\begin{array}{l}0.146^{* * *} \\
0.026\end{array}$ & $\begin{array}{r}-0.004 \\
0.008\end{array}$ & $\begin{array}{l}0.033^{*} \\
0.005\end{array}$ & $\begin{array}{l}0.000 \\
0.011\end{array}$ & $\begin{array}{l}0.126^{* * *} \\
0.149^{* * *}\end{array}$ & $\begin{array}{l}0.096^{* * *} \\
0.015\end{array}$ & $\begin{array}{l}0.013 \\
0.029\end{array}$ & $\begin{array}{l}0.094^{* * * *} \\
0.046^{* * *}\end{array}$ \\
\hline
\end{tabular}

aValues were calculated without hierarchy.

Significance: ${ }^{*} P<0.05,{ }^{* *} P<0.01,{ }^{* * *} P<0.001$.

also a statistically significant location effect within hosts $\left(F_{\mathrm{LH}}=0.030, P<0.0001\right)$. The host effect is statistically significant both in Kilpisjärvi $\left(F_{\mathrm{HK}}=0.094, P<0.0001\right)$ and Abisko $\left(F_{\mathrm{HA}}=0.046, P<0.0001\right)$. These results are also evident in the clustering analysis of females; within Cluster 1, S. phylicifolia + myrsinifolia samples are grouped according to location, but the two $S$. hastata samples form a separate cluster.

The results are not as clear in males in Cluster 1 . The separate $S$. hastata cluster is not present in males, and the overall differentiation is not statistically significant $\left(F_{\mathrm{ST}}=\right.$ $0.105, P=0.079)$. However, within Abisko the overall host 
Host (Location)

a Females

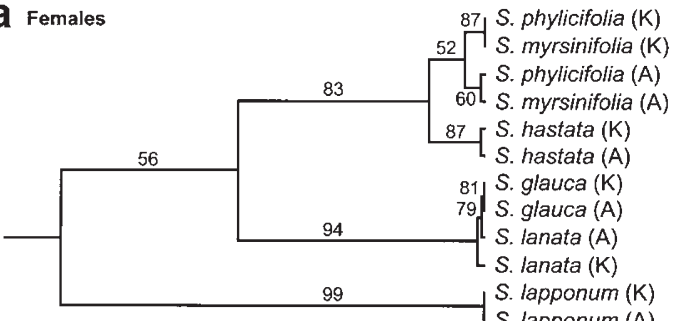

Cluster 1

Cluster 2

Cluster 3
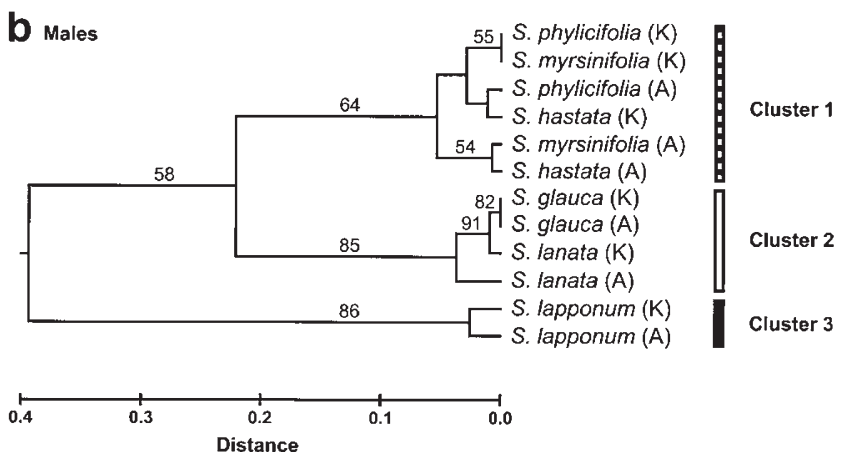

Figure 1 UPGMA clustering dendrograms of Euura mucronata population samples, based on pairwise Nei's (1978) unbiased genetic distances calculated from seven polymorphic allozyme loci. The willow (Salix spp.) hosts and sampling locations are given to the right of the dendrograms. Locations (in parentheses) are abbreviated as follows: $\mathrm{K}=$ Kilpisjärvi, $\mathrm{A}=$ Abisko. Numbers above branches are bootstrap proportions (\%) from 1000 replicates (only values $>50 \%$ shown).

effect is statistically significant $\left(F_{\mathrm{HA}}=0.146, P=0.029\right)$, caused mainly by significant differentiation in the Idh locus. On the other hand, it has to be noted that the power of the tests is probably limited due to the relatively small sample sizes of males (Table 2).

In female Cluster 2, the overall differentiation is statistically significant $\left(F_{\mathrm{ST}}=0.013, P=0.025\right)$, but there is no significant host or location effect (Table 4). There is, however, a significant host within location effect $\left(F_{\mathrm{HL}}=\right.$ $0.009, P=0.020)$, and location within host effect $\left(F_{\mathrm{LH}}=\right.$ $0.015, P=0.038$ ). However, the differentiation is not significant if the analyses are done separately in Kilpisjärvi and Abisko (Table 4).

The overall differentiation is statistically significant also in males within Cluster $2\left(F_{\mathrm{ST}}=0.099, P=0.012\right)$, but there is no significant host nor location effect. The differentiation between hosts is, however, statistically significant within Abisko $\left(F_{\mathrm{HA}}=0.207, P=0.004\right)$, although the only individual locus with significant differentiation is Pgm.

\section{Discussion}

The results clearly demonstrate that in northern Fennoscandia 'E. mucronata' is comprised of at least three separate lineages, one on S. phylicifolia + myrsinifolia + hastata (= Cluster 1 in Figure 1), one on S. lanata + glauca (=Cluster 2) and one on S. lapponum (= Cluster 3). In addition, there may be an additional species within Cluster 1 (on $S$. hastata). This putative species was not found in the male samples but the small sample sizes may be the cause of this. There also seems to be a low level of host-associated genetic differentiation within Cluster 2 .

Clusters 1, 2, and 3 apparently represent 'traditional' E. mucronata, E. lanatae, and E. lappo, respectively (see Malaise, 1920; Zinovjev and Vikberg, 1998; Kopelke, 1999). The morphological inspection revealed that the females reared from S. lanata and S. glauca were of Malaise's (1920) Euura lanatae-type, because all examined individuals had a gradually tapering sawsheath and relatively pale antennae (see also Kopelke, 1999). In contrast, the females reared from the four other willow species had a sawsheath with a broad base that is abruptly constricted to form a sharp apex, and darker antennae. Within this second group, individuals representing different host species could not be consistently separated using Malaise's (1920) description (see also Viitasaari and Vikberg 1985), although the individuals reared from $S$. lapponum are clearly genetically differentiated from the others (Figure 1). Likewise, the inspected males could not be unambiguously grouped according to their host species following Malaise's (1920) morphological descriptions, but genetically the males form the same three main clusters as the females (Figure 1).

Whether the three lineages represent valid biological species (sensu Mayr, 1963), or host races (sensu Diehl and Bush, 1984) remains to be studied. Although there are clear differences in allele frequencies between the three clusters at many loci, no fixed differences could be found. The only exception is the $P g m^{119}$ allele, which is almost diagnostic for individuals collected from S. lapponum $(=$

Table 4 Hierarchical $F$ statistics for each locus and averaged across all loci in female Euura mucronata in Cluster 2 (see Figure 1)

\begin{tabular}{|c|c|c|c|c|c|c|c|c|c|}
\hline & \multicolumn{9}{|c|}{ Locus } \\
\hline & $M e$ & $P g m$ & $M d h-2$ & $T p i$ & $I d h$ & Aat- & 2 & $P g i$ & Total \\
\hline$F_{\mathrm{ST} \text { (OVERALL) }}$ & 0.001 & 0.019 & $0.035^{*}$ & - & -0.002 & 0.013 & & 0.013 & $0.013^{*}$ \\
\hline $\begin{array}{l}F_{\mathrm{HT} \text { (HOSTS) }} \\
F_{\text {LH (LOCATIONS WITHIN HOSTS) }}\end{array}$ & $\begin{array}{r}0.011 \\
-0.006\end{array}$ & $\begin{array}{c}-0.012 \\
0.027^{*}\end{array}$ & $\begin{array}{c}-0.007 \\
0.039 *\end{array}$ & $\begin{array}{l}- \\
-\end{array}$ & $\begin{array}{r}-0.004 \\
0.001\end{array}$ & $\begin{array}{r}-0.002 \\
0.015\end{array}$ & & $\begin{array}{r}-0.011 \\
0.020\end{array}$ & $\begin{array}{r}-0.002 \\
0.015^{*}\end{array}$ \\
\hline $\begin{array}{l}F_{\text {LT (LOCATIONS) }} \\
F_{\text {HL (HOSTS WITHIN LOCATIONS) }}\end{array}$ & $\begin{array}{r}-0.002 \\
0.002\end{array}$ & $\begin{array}{l}0.021 \\
0.006^{*}\end{array}$ & $\begin{array}{l}-0.018 \\
0.046^{*}\end{array}$ & $\begin{array}{l}- \\
-\end{array}$ & $\begin{array}{r}0.007 \\
-0.006\end{array}$ & $\begin{array}{l}0.000 \\
0.013\end{array}$ & & $\begin{array}{l}0.019 \\
0.000\end{array}$ & $\begin{array}{l}0.007 \\
0.009 *\end{array}$ \\
\hline $\begin{array}{l}F_{\text {HK (HOSTS IN KILPISJÄRVI) }}{ }^{a} \\
F_{\text {HA (HOSTS IN ABISKO) }}{ }^{a}\end{array}$ & $\begin{array}{r}-0.001 \\
0.005\end{array}$ & $\begin{array}{r}0.012 \\
-0.002\end{array}$ & $\begin{array}{r}0.076^{*} \\
-0.006\end{array}$ & $\begin{array}{l}- \\
-\end{array}$ & $\begin{array}{c}- \\
-0.007\end{array}$ & $\begin{array}{c}0.013 \\
-\end{array}$ & & $\begin{array}{c}0.000 \\
-\end{array}$ & $\begin{array}{l}0.016 \\
0.000\end{array}$ \\
\hline
\end{tabular}

a Values were calculated without hierarchy.

Significance: ${ }^{*} P<0.05$. 
E. lappo). Thus, occasional hybridization between the putative species cannot be ruled out. The lack of statistically significant deviations from Hardy-Weinberg equilibria indicates that the three species have well-defined host ranges but, on the other hand, the deviations in the S. lanata and S. glauca samples from Kilpisjärvi suggest that there may be some overlap in the oviposition ranges of the species.

Although the phylogenetic relationships of willow species are still poorly known (Argus, 1997; Skvortsov, 1999; Azuma et al, 2000), it is evident that the clustering results (Figure 1) present some clear contradictions compared to the phylogeny of willows. The most evident discrepancy is the combination S. lanata + glauca in Cluster 2 , since these species belong to different subgenera (Skvortsov, 1999; but see Argus, 1997). Instead, S. lanata should be related to $S$. hastata, which, however, is included in Cluster 1 together with S. phylicifolia and S. myrsinifolia. The combination of S. phylicifolia and S. myrsinifolia in Cluster 1 would be expected because of the close relationship between these species (Skvortsov, 1999). On the other hand, S. lapponum is a close relative of S. phylicifolia, yet Cluster $3(=$ E. lappo) is clearly a distinct species of its own. The observed pattern in the feeding ranges obviously precludes parallel cladogenesis between the bud gallers and their willow hosts in the long run and, thus, supports the view that strict parallel cladogenesis between herbivorous insects and plants is a rare exception rather than the rule (Mitter et al, 1991; Farrell and Mitter, 1998).

Chemically, willows are characterized by low-molecular weight phenolic glycosides that occur in different species-specific arrays (Julkunen-Tiitto, 1989) and appear to govern the food selection and/or oviposition preferences of many willow-feeding insects (Pasteels and Rowell-Rahier, 1992; Kolehmainen et al, 1994, 1995; Roininen et al, 1999). Julkunen-Tiitto (1989) quantified the levels of simple phenolic glycosides in northern willows. According to her results, S. lanata and S. glauca are rather similar in their overall chemistry, which could explain their association in Cluster 2. Also S. myrsinifolia and S. hastata are chemically relatively close but, in contrast, S. phylicifolia is chemically more similar to $S$. lapponum than to either of them. Thus, the question becomes, why is Cluster $1(=$ E. mucronata) associated with S. phylicifolia + myrsinifolia + hastata, rather than with S. phylicifolia + lapponum?

These results concerning the relationship between willow chemistry and bud galler host associations should, however, be treated with caution. As Nyman and Julkunen-Tiitto (2000) showed, the galls induced by six leafgalling Pontania species are chemically very different from the respective host species. Consequently, it is also very likely that the chemistry of bud galls may have little to do with host chemistry. Host chemistry can, of course, have an effect via the behaviour of the ovipositing females that use chemical cues for selecting appropriate hosts. The host-identification process, in turn, may be governed by only a few compounds: tremulacin stimulates oviposition of females in the stem galler E. lasiolepis Smith (Roininen et al, 1999), and in the case of the stem galler E. amerinae $\mathrm{L}$. the main stimulant seems to be $2^{\prime}-$ O-acetylsalicortin (Kolehmainen et al, 1994). It should also be noted that willow chemistry (and phylogeny) seems to have only a minor role in the evolution of host use in the nematine gallers as a whole (Nyman et al, 2000), and similar results were also found by Roininen et al (1993) in the stem-galling E. atra complex.

Furthermore, the host associations apparently cannot be explained by simple allopatric speciation, because the host willows in this study are largely sympatric. According to Skvortsov (1999), the host species can be divided into two main groups based on their geographic distribution: S. hastata, S. lanata, and S. glauca occur throughout the northern reaches of the Palaearctic region, whereas $S$. myrsinifolia, S. phylicifolia, and S. lapponum occur mainly in the European parts of Russia, in Fennoscandia, and in the northern parts of Central Europe. There is considerable distributional overlap both within and between these groups, and although the hosts differ somewhat in their habitat, there is also extensive overlap. Further studies are needed about the ranges of these host species during the Pleistocene glaciations, that may have created separate populations.

It is likely that the pattern of host use by the bud gallers is connected to phenological or ecological differences between the host species, but these may be more difficult to analyse. Phenological differences between willow species certainly exist, but there is currently no data available on this. The existence of such differences is suggested by the fact that the individuals reared from S. lanata and S. glauca tended to emerge earlier than the ones reared from the other willow species (Nyman, 2000). However, the possibility for phenological (allochronic) differentiation may be counteracted by the fact that the oviposition period of E. mucronata lasts for several weeks, because buds become available over an extended period of time as the shoot grows (Roininen, 1991). Another possibility is that there are morphological differences between willows: the bud gallers oviposit through the petiole base into developing buds (Kopelke, 1998) and it is possible that ovipositor length may restrict the suitability of hosts.

In conclusion, it is evident that in northern Fennoscandia 'E. mucronata' is not a single polyphagous species, but is instead a complex consisting of several mono- or oligophagous sibling species or host races; determining the exact status of these genetically differentiated lineages will require further study. According to the present results, no simple explanation, such as host phylogeny or overall chemistry, can explain the host associations of the bud gallers. Instead, the pattern observed in 'E. mucron$a t a^{\prime}$ suggests that these sawflies may be constrained by the ecological, morphological, and/or phenological characteristics of the host plants. Finally, it should be remembered that in addition to the six Fennoscandian host species included in this study, bud galls can be found on numerous other willow species in other parts of the Holarctic region, so it is likely that additional cryptic bud-galling species exist.

\section{Acknowledgements}

I wish to thank J Tahvanainen, H Roininen, L Sundström, P Pamilo, L Excoffier, and two anonymous reviewers for their suggestions concerning data analysis and/or comments on the manuscript. Funding for this study was provided by the Emil Aaltonen Foundation, and the University of Joensuu Faculty of Mathematics and Natural Sciences. 


\section{References}

Argus GW (1997). Infrageneric classification of the genus Salix (Salicaceae) in the New World. Syst Bot Monogr 52: 1-121.

Azuma T, Kajita T, Yokoyama J, Ohashi H (2000). Phylogenetic relationships of Salix (Salicaceae) based on $r b c L$ sequence data. Am J Bot 87: 67-75.

Becerra JX (1997). Insects on plants: macroevolutionary chemical trends in host use. Science 276: 253-256.

Benson BR (1958). Hymenoptera 2. Symphyta. Handbooks for the Identification of British Insects VI(2c): 139-252.

Bernays E, Graham M (1988). On the evolution of host specificity in phytophagous arthropods. Ecology 69: 886-892.

Berlocher SH (1999). Host race or species? Allozyme characterization of the 'flowering dogwood fly', a member of the Rhagoletis pomonella complex. Heredity 83: 652-662.

Butlin RK (1990). Divergence in emergence time of host races due to differential gene flow. Heredity 65: 47-50.

Condon MA, Steck GJ (1997). Evolution of host use in fruit flies of the genus Blepharoneura (Diptera: Tephritidae): cryptic species on sexually dimorphic host plants. Biol J Linn Soc 60: 443-466.

Crespi BJ, Sandoval CP (2000). Phylogenetic evidence for the evolution of ecological specialization in Timema walkingsticks. J Evol Biol 13: 249-262.

Diehl SR, Bush GL (1984). An evolutionary and applied perspective of insect biotypes. Annu Rev Entomol 29: 471-504.

Ehrlich PR, Raven PH (1964). Butterflies and plants: a study in coevolution. Evolution 18: 586-608.

Emelianov I, Mallet J, Baltensweiler W (1995). Genetic differentiation in Zeiraphera diniana (Lepidoptera: Tortricidae, the larch budmoth): polymorphism, host races or sibling species? Heredity 75: 416-424.

Excoffier L, Smouse PE, Quattro JM (1992). Analysis of molecular variance inferred from metric distances among DNA haplotypes: application to human mitochondrial DNA restriction data. Genetics 131: 479-491.

Farrell BD, Mitter C (1998). The timing of insect/plant diversification: might Tetraopes (Coleoptera: Cerambycidae) and Asclepias (Asclepiadaceae) have co-evolved? Biol J Linn Soc 63: 553-577.

Ferguson A (1980). Biochemical Systematics and Evolution. Blackie: Glasgow.

Filchak KE, Roethele JB, Feder JL (2000). Natural selection and sympatric divergence in the apple maggot Rhagoletis pomonella. Nature 407: 739-742.

Futuyma DJ, Keese MC (1992). Evolution and coevolution of plants and phytophagous arthropods. In: Rosenthal GA, Berenbaum MR (eds) Herbivores: Their Interactions with Secondary Plant Metabolites, 2nd edn, Vol. II, Ecological and Evolutionary Processes, Academic Press: New York. pp. 439-475.

Gauld I, Bolton B (1988). The Hymenoptera. Oxford University Press: Oxford.

Groman JD, Pellmyr O (2000). Rapid evolution and specialization following host colonization in a yucca moth. J Evol Biol 13: 223-236.

Guo SW, Thompson EA (1992). Performing the exact test of Hardy-Weinberg proportion for multiple alleles. Biometrics 48 : 361-372.

Hartley SE (1992). The insect galls on willow. Proc R Soc Edinb 98B: 91-104.

Herbst J, Heitland W (1994). Genetic differentiation among populations of the sawfly-species Platycampus luridiventris, associated with different Alder species (Hymenoptera: Tenthredinidae). Entomol Gener 19: 39-48.

Janz N, Nylin S (1997). The role of female search behaviour in determining host plant range in plant feeding insects: a test of the information processing hypothesis. Proc $R$ Soc Lond B 264: 701-707.

Jermy T (1984). Evolution of insect/host plant relationships. Am Nat 124: 609-630.

Julkunen-Tiitto R (1989). Phenolic constituents of Salix: a chemo- taxonomic survey of further Finnish species. Phytochemistry 28: 2115-2125.

Kolehmainen J, Roininen H, Julkunen-Tiitto R, Tahvanainen J (1994). Importance of phenolic glucosides in host selection of shoot galling sawfly, Euura amerinae, on Salix pentandra. J Chem Ecol 20: 2455-2466.

Kolehmainen J, Julkunen-Tiitto R, Roininen H, Tahvanainen J (1995). Phenolic glucosides as feeding cues for willow-feeding leaf beetles. Entomol Exp Appl 74: 235-243.

Kopelke J-P (1996). Die Euura atra- und amerinae-Gruppe in Nord- und Mitteleuropa (Insecta: Hymenoptera: Tenthredinidae: Nematinae). Senckenb Biol 76: 93-113.

Kopelke J-P (1998). Eiablage-Strategien bei gallenbildenden Arten der Blattwespen-Gattungen Pontania, Euura und Phyllocolpa (Hymenoptera: Tenthredinidae: Nematinae). Entomol Gener 22: 251-275.

Kopelke J-P (1999). Gallenerzeugende Blattwespen Europas Taxonomische Grundlagen, Biologie und Ökologie (Tenthredinidae: Nematinae: Euura, Phyllocolpa, Pontania). Cour Forsch-Inst Senckenb 212: 1-183.

Levins R, MacArthur R (1969). An hypothesis to explain the incidence of monophagy. Ecology 50: 910-911.

Malaise R (1920). Beiträge zur Kenntnis swedischer Blattwespen. Entomol Tidskr 40: 97-128.

Mayr E (1963). Animal Species and Evolution. Harvard University Press: Cambridge.

Mardulyn P, Milinkovitch MC, Pasteels JM (1997). Phylogenetic analyses of DNA and allozyme data suggest that Gonioctena leaf beetles (Coleoptera; Chrysomelidae) experienced convergent evolution in their history of host-plant family shifts. Syst Biol 46: 722-747.

Miller MP (1997). Tools for population genetics analyses (TFPGA) 1.3: A Windows program for the analysis of allozyme and molecular population genetic data. Computer software distributed by author.

Mitter C, Brooks DR (1983). Phylogenetic aspects of coevolution. In: Futuyma DJ, Slatkin M (eds) Coevolution, Sinauer: Sunderland. pp. 65-98.

Mitter C, Farrell B, Futuyma DJ (1991). Phylogenetic studies of insect-plant interactions: insights into the genesis of diversity. Trends Ecol Evol 6: 290-293.

Nei M (1978). Estimation of average heterozygosity and genetic distance from a small number of individuals. Genetics 89: 583-590.

Nyman T (2000). Phylogeny and ecological evolution of gallinducing sawflies (Hymenoptera: Tenthredinidae). University of Joensuu, Ph.D. Dissertations in Biology 6: 1-92.

Nyman T, Julkunen-Tiitto R (2000). Manipulation of the phenolic chemistry of willows by gall-inducing sawflies. Proc Natl Acad Sci USA 97: 13184-13187.

Nyman T, Roininen H, Vuorinen JA (1998). Evolution of different gall types in willow-feeding sawflies (Hymenoptera: Tenthredinidae). Evolution 52: 465-474.

Nyman T, Widmer A, Roininen H (2000). Evolution of gall morphology and host-plant relationships in willow-feeding sawflies (Hymenoptera: Tenthredinidae). Evolution 54: 526-533.

Pasteels JM, Rowell-Rahier M (1992). The chemical ecology of herbivory on willows. Proc $R$ Soc Edinb 98B: 63-73.

Price PW, Roininen H (1993). Adaptive radiation in gall induction. In: Wagner M, Raffa KF (eds) Sawfly Life History Adaptations to Woody Plants, Academic Press: New York. pp 229257.

Reynolds J, Weir BD, Cockerham CC (1983). Estimation of the coancestry coefficient: basis for a short-term genetic distance. Genetics 105: 767-779.

Rice WR (1989). Analyzing tables of statistical tests. Evolution 43: 223-225.

Roininen H (1991). Temporal change in the location of egg-laying by a bud-galling sawfly, Euura mucronata, on growing shoots of Salix cinerea. Oecologia 87: 265-269.

Roininen H, Vuorinen J, Tahvanainen J, Julkunen-Tiitto, R 
(1993). Host preference and allozyme differentiation in shoot galling sawfly, Euura atra. Evolution 47: 300-308.

Roininen H, Price PW, Julkunen-Tiitto R, Tahvanainen J, Ikonen A (1999). Oviposition stimulant for a gall-inducing sawfly, Euura lasiolepis, on willow is a phenolic glucoside. J Chem Ecol 25: 943-953.

Schneider S, Roessli D, Excoffier L (2000). ARLEQUIN version 2.000. A software for population genetics data analysis. Genetics and Biometry Laboratory, University of Geneva, Switzerland.

Skvortsov AK (1999). Willows of Russia and adjacent countries. Taxonomical and geographical revision. University of Joensuu Faculty of Mathematics and Natural Sciences Report Series 39: $1-307$.

Smith EL (1970). Biosystematics and morphology of Symphyta. II. Biology of gall-making nematine sawflies of the California region. Ann Entomol Soc Am 63: 35-51.

Smith DC (1988). Heritable divergence of Rhagoletis pomonella host races by seasonal asynchrony. Nature 336: 66-67.
Sturgeon KB, Mitton JB (1986). Allozyme and morphological differentiation of mountain pine beetles Dendroctonus ponderosae Hopkins (Coleoptera: Scolytidae) associated with host tree. Evolution 40: 290-302.

Thompson JN (1994). The Coevolutionary Process. University of Chicago Press: Chicago.

Viitasaari M, Vikberg V (1985). A checklist of the sawflies (Hymenoptera, Symphyta) of Finland. Not Entomol 65: 1-17.

Vuorinen J (1984). Electrophoretic expression of genetic variation and duplicate gene activity in vendace, Coregonus albula (Salmonidae). Hereditas 101: 85-96.

Weir BS, Cockerham CC (1984). Estimating F-statistics for the analysis of population structure. Evolution 38: 1358-1370.

Wright S (1978). Evolution and the Genetics of Populations, Vol. 4. Variability Within and Among Natural Populations. University of Chicago Press: Chicago.

Zinovjev AG, Vikberg V (1998). On the biology of Nematinae with hiding larvae (Hymenoptera, Symphyta, Tenthredinidae). Beitr Ent 48: 145-155. 